\title{
A potential prognostic model based on miRNA expression profile in The Cancer Genome Atlas for bladder cancer patients
}

\author{
Yan Liu', Dong Yan Zhu ${ }^{1}$, Hong Jian Xing ${ }^{3}$, Yi Hou $^{4}$ and Yan Sun ${ }^{1 *}$
}

\begin{abstract}
Background: This study aimed to construct prognostic model by screening prognostic miRNA signature of bladder cancer.

Methods: The miRNA expression profile data of bladder cancer (BC) in The Cancer Genome Atlas (TCGA) were obtained and randomly divided into the training set and the validation set. Differentially expressed miRNAs (DEMs) between $\mathrm{BC}$ and normal control samples in the training set were firstly identified, and DEMs related to prognosis were screened by Cox Regression analysis. Then, the MiR Score system was constructed using X-Tile based cutoff points and verified in the validation set. The prognostic clinical factors are selected out by univariate and multivariate Cox Regression analysis. Finally, the mRNAs related to prognosis were screened and the biological pathway analysis was carried out.

Results: We identified the 7-miRNA signature was significantly associated with the patient's Overall Survival (OS). A prognostic model was constructed based on the prognostic 7-miRNA signature, and possessed a relative satisfying predicted ability both in the training set and validation set. In addition, univariate and multivariate Cox Regression analysis showed that age, lymphovascular invasion and MiR Score were considered as independent prognostic factors in BC patients. Furthermore, based on MiR Score prognostic model, several differentially expressed genes (DEGs), such as WISP3 and UNC5C, as well as their related biological pathway(s), including cell-cell adhesion and neuroactive ligand-receptor interaction, were considered to be related to BC prognosis.
\end{abstract}

Conclusion: The prognostic model which was constructed based on the prognostic 7-miRNA signature presented a high predictive ability for BC.

Keywords: Prognostic model, Bladder cancer, The Cancer Genome Atlas

\section{Background}

Bladder cancer (BC) is one of the most common malignant tumor of the urinary system, which is characterized by the high rate of non-muscle invasive BC (NMIBC) at the moment of diagnosis $(75-80 \%)[1,2]$, with approximately 3.4 million affected cases and 188,000 deaths in

\footnotetext{
*Correspondence: sunyansun_8@163.com

1 Anesthesiology Department, Jilin Univ, China Japan Union Hosp, 126 Xiantai St, Changchun 130033, Jilin, People's Republic of China

Full list of author information is available at the end of the article
}

2015 [3]. Currently, the standard BC screening methods include cystoscopy, sonography, and urinary cytology; however, its high invasiveness and low accuracy still cannot be neglected [4]. Although current treatments have improved 5-year survival rate of $\mathrm{BC}$, most patients have delayed diagnosis of proximal and distal metastasis due to the atypical symptoms of early $\mathrm{BC}$, resulting in poor treatment efficacy and prognosis. Radical cystectomy $(\mathrm{RC})$ is usually performed in patients with early diagnosis of muscular invasive cystitis (MIBC). This is not the best solution because patients have a poor quality of life after

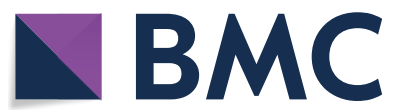

(c) The Author(s) 2020. This article is licensed under a Creative Commons Attribution 4.0 International License, which permits use, sharing, adaptation, distribution and reproduction in any medium or format, as long as you give appropriate credit to the original author(s) and the source, provide a link to the Creative Commons licence, and indicate if changes were made. The images or other third party material in this article are included in the article's Creative Commons licence, unless indicated otherwise in a credit line to the material. If material is not included in the article's Creative Commons licence and your intended use is not permitted by statutory regulation or exceeds the permitted use, you will need to obtain permission directly from the copyright holder. To view a copy of this licence, visit http://creativeco mmons.org/licenses/by/4.0/. The Creative Commons Public Domain Dedication waiver (http://creativecommons.org/publicdomain/ zero/1.0/) applies to the data made available in this article, unless otherwise stated in a credit line to the data. 
surgery and a high rate of recurrence and mortality in the short time after surgery [5]. As far as patients with BC are concerned, it is impossible to predict which of them will have disease progression. Therefore, it is important to further reveal novel diagnostic and therapeutic methods, as well as underlying risk factors for poor prognosis of $B C$ patients.

MiRNAs (miRNAs) are endogenous non-coding singlestranded small RNA molecules that regulate gene expression by repressing translational efficiency or decreasing target mRNA stability, thereby participating in various key cell biological processes, such as embryonic development, tumor cell proliferation, differentiation, and apoptosis [6, 7]. MiRNAs are known to be dysregulated in $\mathrm{BC}$ and implicated in the pathogenesis of bladder tumors mainly through their effect on genes involved in two molecular pathways, specifically the gene which codes tumor protein 53 (TP53) [8] and fibroblast growth factor receptor 3 (FGFR3) [9]. Previous studies have reported that the dysregulation of miRNAs is related to the prognosis and the progression of $\mathrm{BC}[1]$, such as miR144-5p [10], miR-199 family [11], and miR-214 [12]. For example, Falzone et al. [13] reported that downregulated hsa-miR-145-5p and hsa-miR-214-3p may modulate the expression of both EMT and NGAL/MMP-9 pathways in BC using bioinformatic analysis. Accordingly, miRNAs may be considered good candidates as biomarkers for both prognosis and diagnosis of BC. Hence, miRNAs profiling studies from different tissues represent an excellent alternative application for these short sequences as biomarkers with clinical significance. It is widely known that poor prognosis as a major challenge for the treatment of $\mathrm{BC}$ leads to a low survival rate of $\mathrm{BC}$ patients, and gene mutation and environment exposure have been identified to be associated with this outcome. To our knowledge, however, a prognostic model of $\mathrm{BC}$ is rarely reported. In the current study, we aimed to develop a prognostic model for $\mathrm{BC}$ that provides some useful insights in improving the prognosis of $\mathrm{BC}$ patients and helps to increase their overall survival. For this purpose, the miRNA expression profile data of $\mathrm{BC}$ based on The Cancer Genome Atlas (TCGA) were analyzed to screen miRNAs related to $\mathrm{BC}$ prognosis and then construct a $\mathrm{BC}$ prognostic model using bioinformatic methods. In addition, the related clinical prognostic factors, messenger RNAs (mRNAs) and biological pathways were analyzed based on this model.

\section{Methods}

\section{Data extraction and grouping}

The clinical information, the miRNA expression profile and the mRNA data based on the Illumina HiSeq2000 RNA Sequencing (Illumina, San Diego, CA, USA) platform of a total of 432 samples of BC patients were downloaded from TCGA (https://gdc-portal.nci.nih.gov/) [14]. After corresponding to clinical information of them, a total of 428 samples with corresponding information were included in our study, of which 409 were tumor samples (BC group) and 19 were normal control samples (control group). Then, $\mathrm{BC}$ group were randomly divided into two groups: 204 tumor samples utilized as the training set and 205 tumor samples utilized as the validation set. The clinical information of tumor samples in the training set and validation set are shown in Table 1.

\section{DEMs screening in the training set}

First, miRNAs with median value as 0 in the training set were removed, which means the read counts obtained through the Illumina Hiseq. Next, based on the expression information provided by TCGA, limma package

Table 1 The clinical information of bladder cancer tumor samples in the training set and validation set

\begin{tabular}{lll}
\hline Clinical characteristics & Training set (N=204) & Validation set (N=205) \\
\hline Age (years, mean \pm SD) & $68.46 \pm 27.57$ & $67.69 \pm 9.86$ \\
Gender (male/female) & $154 / 50$ & $148 / 57$ \\
Pathologic M (M0/M1/-) & $99 / 7 / 98$ & $96 / 4 / 105$ \\
Pathologic N (NO/N1/N2/N3/-) & $122 / 23 / 36 / 2 / 21$ & $115 / 23 / 40 / 6 / 21$ \\
Pathologic T (T1/T2/T3/T4/-) & $3 / 65 / 95 / 27 / 14$ & $1 / 55 / 99 / 31 / 19$ \\
Pathologic stage (I/II/II/IV/-) & $2 / 71 / 65 / 65 / 1$ & $0 / 60 / 74 / 70$ \\
Pathologic grade (high/low/-) & $194 / 9 / 1$ & $191 / 12 / 2$ \\
Radiotherapy (yes/no/-) & $6 / 186 / 12$ & $14 / 177 / 14$ \\
Lymphovascular invasion (yes/no/-) & $65 / 73 / 66$ & $85 / 59 / 61$ \\
Recurrence (yes/no) & $44 / 126 / 34$ & $43 / 124 / 38$ \\
Dead (death/alive/-) & $92 / 110 / 2$ & $86 / 117 / 2$ \\
Overall survival time (months, mean \pm SD) & $26.76 \pm 27.57$ & $27.45 \pm 28.19$
\end{tabular}

$M$ distant metastases, $N$ regional lymph node, $T$ tumor size 
(version 3.34.7, https://bioconductor.org/packages/relea $\mathrm{se} / \mathrm{bioc} / \mathrm{html} /$ limma.html) [15] in $\mathrm{R}$ 3.4.1 was used to screen DEMs between $\mathrm{BC}$ and normal control samples with the thresholds of false discovery rate $(\mathrm{FDR})<0.05$ and $\mid \log$ fold change $(\mathrm{FC}) \mid>1$. At last, according to the expression values of DEMs in the training set, bidirectional hierarchical clustering based on centered Pearson correlation algorithm was performed by pheatmap (version 1.0.8, https://cran.r-project.org/web/packages/pheat map/index.html) [16] in R 3.4.1.

\section{DEMs screening related to prognosis}

Combined with the clinical prognostic information of $\mathrm{BC}$ samples in the training set and the expression levels of DEMs, DEMs related to the OS were screened by Univariate Cox Regression analysis survival package (version 2.41.3, https://cran.r-project.org/web/packages/survival/ index.html) [17] in R 3.4.1, with the threshold of log-rank $p$-value $<0.05$.

\section{Construction and verification of prognosis risk assessment model based on DEMs levels}

Based on DEMs related to prognosis, the optimized DEMs signature was screened using LASSO Cox Regression model [18] of penalized package (version 0.9.50, https://cran.r-project.org/web/packages/penalized/index .html) [19] in R 3.4.1. The optimized parameter lambda in this model was obtained by 1000 cycles calculation of cross-validation likelihood (cvl) algorithm. Subsequently, the cutoff value of the optimized DEM signature was calculated using X-Tile Bio-Informatics Tool (https ://medicine.yale.edu/lab/rimm/research/software.aspx) [20] with the threshold of Monte-Carlo $p$-value $<0.05$. The sample status was defined according to the cutoff value of each miRNA: status $=1$ when the expression level of miRNA $>$ the cutoff value; while status $=0$ when the expression level of miRNA $<$ the cutoff value [21]. After that, the risk assessment model (MiR score) was constructed for each sample by the linear combination of miRNA status weighted by regression coefficient as follows: MiR Score $=\sum \beta_{\text {miRnA }} \times$ Status $_{\text {miRna } n}$. The $\beta$ represented prognostic regression coefficient and status was defined as previously mentioned. We found that technical biases of miRNA data from the TCGA are not affecting the differential expression due to the presence of sequencing procedures or batches. According to the median value of MiR Score, all samples in the training set were divided into high risk and low risk groups. The Kaplan-Meier (K-M) survival curve analysis was used to estimate the prognosis difference between high risk and low risk groups. Meanwhile, Area Under Receiver Operating Characteristic (AUROC) curve analysis was used to assess the prognostic model. Similar to the above, all samples in the validation set were divided into high risk and low risk groups, and this model was further verified in the validation set and assessed using $\mathrm{K}-\mathrm{M}$ survival analysis and Receiver Operating Characteristic (ROC) curve.

\section{Further analysis of the prognostic factors}

The independent prognostic factors based on the clinical information of tumor samples in the training set and validation set were analyzed using univariate and multivariate Cox Regression analysis survival package (version 2.41.1) in R 3.4.1. Based on these independent prognostic factors, the nomogram of 3- and 5-year survival prediction models were constructed using rms package (version 5.1-2, https://cran.r-project.org/web/packages/rms/ index.html) in R 3.4.1.

\section{The screening of mRNA related to prognosis and pathway analysis}

The RNA-seq profile data of $\mathrm{BC}$ which paired with miRNA expression profile data were extracted, and then divided into high risk and low risk groups according to MiR Score. Next, differentially expressed genes (DEGs) between high risk and low risk groups were screened using limma package with the thresholds of FDR $<0.05$ and $\log$ FC $>1$. Following this, Gene Ontology (GO) functional annotation associated with biological process analysis [22] and Kyoto Encyclopedia of Genes and Genomes (KEGG) pathway enrichment analysis [23] were carried out using Database for Annotation, Visualization and Integrated Discovery (DAVID) program (v 6.8, https:// david.ncifcrf.gov/) [24]. The $p$ value $<0.05$ was considered as the cutoffs for significantly statistical difference in functional analyses.

\section{Results}

\section{DEMs screening in the training set}

Among all samples contained in the training set, miRNAs with median value of read counts as 0 , which indicate not expressed miRNAs, were filtered (Fig. 1a). Based on the selective criteria, a total of 134 DEMs were identified between BC and normal control samples, including 18 downregulated and 116 upregulated miRNAs (Fig. 1b). Then, bidirectional hierachical clustering was conducted for these 134 DEMs, indicating that these identified DEMs could significantly distinct tumor samples from the normal controls (Fig. 1c).

\section{Construction and verification of prognostic models}

Among 204 BC samples in the training set, survival prognostic information was recorded in 202 BC samples. Based on the above 134 DEMs between BC and controls, univariate Cox Regression analyses were performed, and 
a
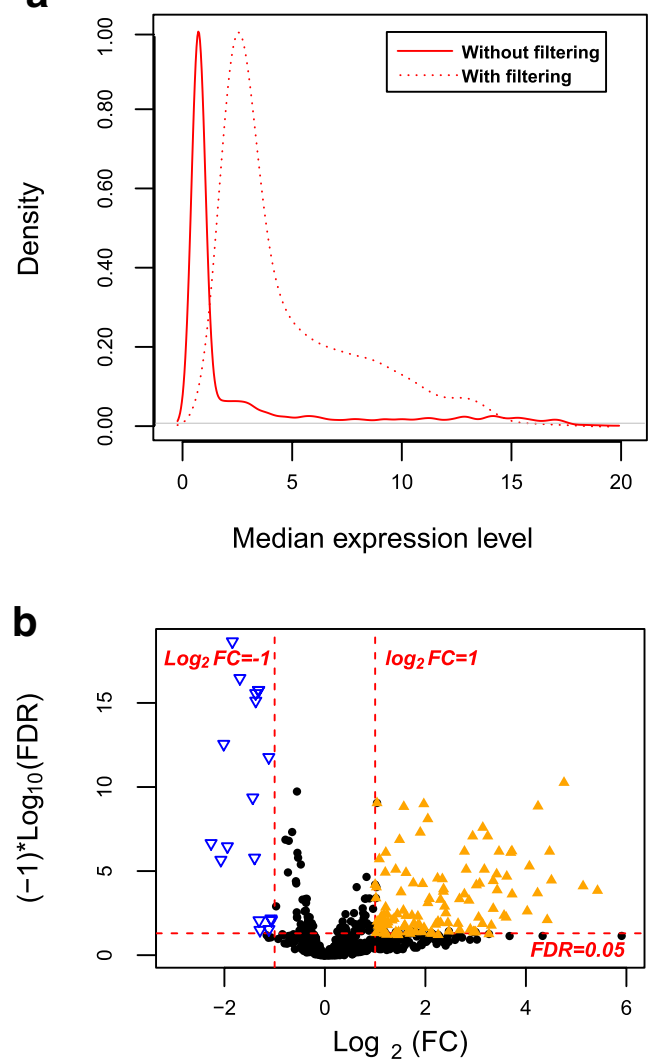

c

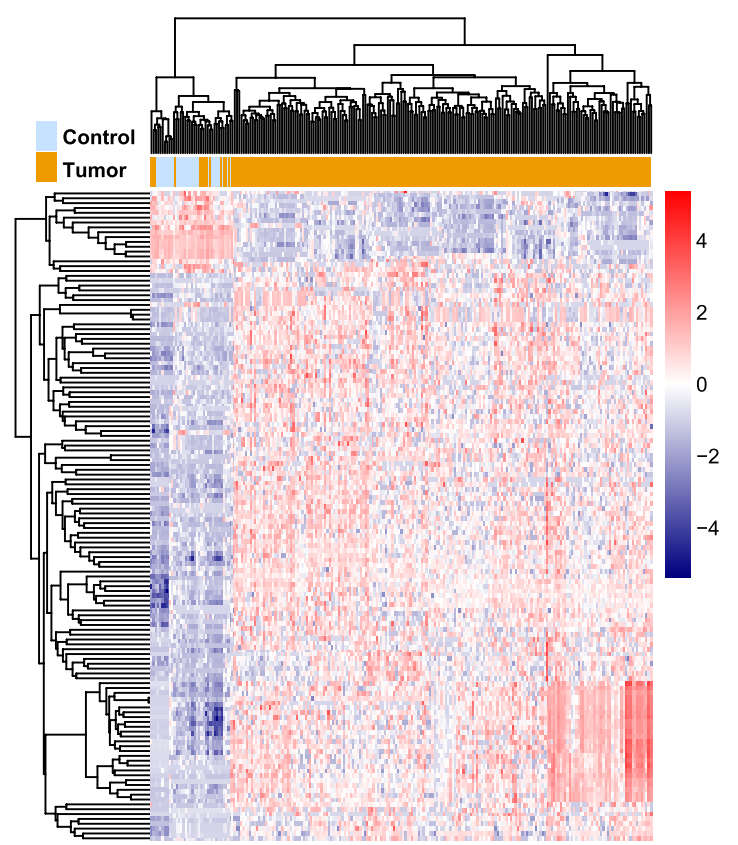

Fig. 1 a The density distribution curve of miRNA expression values before and after filtering. $\mathbf{b}$ Volcano map. Orange triangle, blue triangle, and black dots indicate genes are up-regulated, down-regulated, and non-significant differentially expressed miRNAs, respectively. c A bidirectional hierarchical clustering map based on 134 DEGs. Blue and orange sample bars represent normal control samples and tumor samples

then a total of 21 DEMs were significantly associated with the patient's OS. Based on these 21 DEMs, the optimized DEMs signatures were screened by LASSO Cox Regression model. After 1000 cycles calculation of cvl algorithm, lambda was confirmed as 11.567 and the maximum value of cvl was -509.633 (Fig. 2a). As a result, 7 DEMs, including hsa-miR-1247, hsa-miR-1304, hsamiR-1911, hsa-miR-204, hsa-miR-33b, hsa-miR-3934, and hsa-miR-526b, were obtained (Fig. 2b, Table 2). The cutoff value of these 7 DEMs was calculated using X-Tile Bio-Informatics Tool (Table 2).

According to the miRNA status, the MiR Score risk assessment model was constructed as follows: MiR Score $=(0.0331) \times$ Status $_{\text {hsa-mir-1247 }}+(0.0375) \times$ Status $_{\text {hsa- }}$ mir-1304 $+(0.1274) \times$ Status $_{\text {hsa-mir-1911 }}+(0.1443) \times$ Status $_{\text {hsa- }}$ mir-204 $+(0.2346) \times$ Status $_{\text {hsa-mir-33b }}+(-0.1378) \times$ Status $_{\text {hsa-mir-3934 }}+(0.0662) \times$ Status $_{\text {hsa-mir-526b }}$ The distribution of the MiR Score in the training set and validation set are shown in Fig. 3a left and b left, respectively. ROC curve analysis revealed that the area under curve
(AUC) of 3- and 5-year survival were 0.781 and 0.778 in the training set, as well as 0.781 and 0.762 in the validation set, respectively, indicating that this model possessed a relative satisfying predicted ability both in the training set and validation set (Fig. 3a, b middle). Meanwhile, the estimation of $\mathrm{K}-\mathrm{M}$ survival analysis showed that the OS of patients in the low-risk group was significantly longer than that in the high-risk group $\left(p=1.905 \times 10^{-10}\right.$, and $p=8.821 \times 10^{-3}$, Fig. 3a, b right) in the training set and validation set, respectively.

\section{The independent prognostic factors analysis}

Univariate and multivariate Cox Regression analysis showed that age $>65$ years [hazard ratio $=1.016,95 \%$ confidence interval (CI) 1.011-1.430, $p=2.034 \times 10^{-2}$ and $\mathrm{HR}=1.069,95 \%$ CI $\left.1.0262-1.113, p=1.340 \times 10^{-3}\right]$, lymphovascular invasion $(\mathrm{HR}=1.709,95 \% \mathrm{CI} 0.893-$ $3.274, p=1.050 \times 10^{-2}$ and $\mathrm{HR}=1.338$, 95\% CI $1.056-$ $\left.3.177, p=4.509 \times 10^{-2}\right)$ and MiR Score $(\mathrm{HR}=3.431,95 \%$ CI $1.919-6.130, p=3.180 \times 10^{-5}$ and $\mathrm{HR}=2.353,95 \% \mathrm{CI}$ 

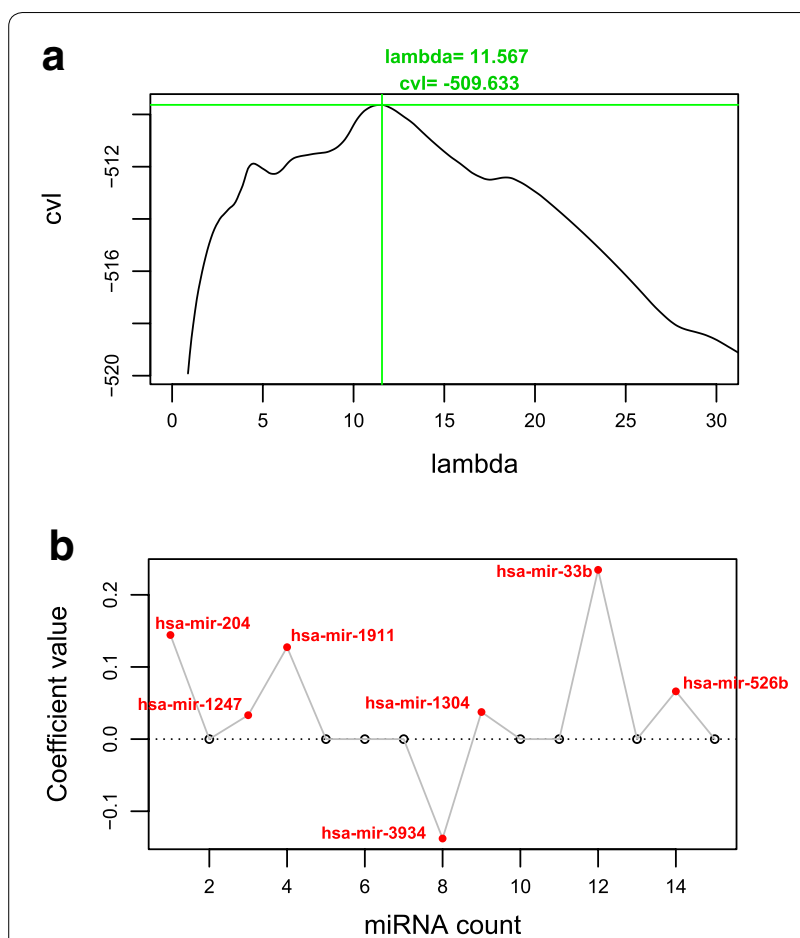

Fig. 2 a The lambda parametric curves by cross-validation likelihood (cvl) algorithm. Horizontal axis and vertical axis represent lambda and $\mathrm{cVl}$, respectively; the intersection of green dotted line represent that the maximum value of $\mathrm{cvl}$ was -509.633 when lambda was 11.567 . b Coefficient distribution diagram of the optimized seven DEMs related to prognosis based on LASSO Cox Regression model

Table 2 The optimized seven differentially expressed miRNAs related to prognosis in bladder cancer

\begin{tabular}{|c|c|c|c|c|c|}
\hline miRNA_ID & $\beta$ & HR & $95 \% \mathrm{Cl}$ & $p$-value & X-tile cutoff \\
\hline $\begin{array}{l}\text { hsa- } \\
\text { miR-1247 }\end{array}$ & 0.0331 & 1.0282 & $\begin{array}{l}0.9199- \\
1.1492\end{array}$ & 0.0062 & 2.76 \\
\hline $\begin{array}{l}\text { hsa- } \\
\text { miR-1304 }\end{array}$ & 0.0375 & 1.2148 & $\begin{array}{l}0.9590- \\
1.5388\end{array}$ & 0.0107 & 1.91 \\
\hline $\begin{array}{l}\text { hsa- } \\
\text { miR-1911 }\end{array}$ & 0.1274 & 1.2425 & $\begin{array}{c}1.0912- \\
1.4146\end{array}$ & 0.0010 & 2.42 \\
\hline hsa-miR-204 & 0.1443 & 1.2205 & $\begin{array}{c}1.0321- \\
1.4432\end{array}$ & 0.0198 & 0.96 \\
\hline hsa-miR-33b & 0.2346 & 1.2055 & $\begin{array}{c}0.8228- \\
1.7662\end{array}$ & 0.0338 & 2.78 \\
\hline $\begin{array}{l}\text { hsa- } \\
\text { miR-3934 }\end{array}$ & -0.1378 & 0.6204 & $\begin{array}{c}0.4611- \\
0.8349\end{array}$ & 0.0016 & 1.09 \\
\hline $\begin{array}{l}\text { hsa-miR- } \\
526 b\end{array}$ & 0.0662 & 1.4881 & $\begin{array}{r}0.9502- \\
2.3303\end{array}$ & 0.0424 & 4.74 \\
\hline
\end{tabular}

$\beta$ prognostic regression coefficient; $H R$ hazard ratio

1.0702-5.176, $p=3.328 \times 10^{-2}$ ) were considered as the independent prognostic factors in $\mathrm{BC}$ patients both in the training set and validation set, respectively (Table 3 ). Furthermore, the nomogram of 3- and 5-year survival prediction models of these independent prognostic factors were constructed as Fig. 4a. The nomogram of 5-year survival prediction showed compliance to actual 5-year survival (Fig. 4b).

\section{Functional enrichment analysis of DEGs related to prognosis}

Based on the selective criteria, a total of 389 DEGs were identified between high risk and low risk groups, including 33 downregulated and 356 upregulated genes, in which several DEGs, such as WISP3 and UNC5C were related to biological pathway (Fig. 5a). WISP3 and UNC5C are the significant DEGs between high risk and low risk group. Then, clustering analysis for these DEGs was conducted, indicating that these identified DEGs could significantly distinct high risk from low risk groups (Fig. 5b). To further identify the functional characteristics of DEGs, the functional enrichment analyses of genes were conducted with DAVID. Consequently, the Biology Process (BP) analysis of DEGs revealed that the significant enriched terms primarily concentrated on ion transport, cell-cell adhesion, neurological system process, metal ion transport, cell-cell signaling, cation transport, transmission of nerve impulse, neuron differentiation, synaptic transmission, muscle contraction, and cell morphogenesis involved in neuron differentiation (Fig. 5c). In addition, the KEGG pathway analysis implied that these DEGs were responsible for neuroactive ligand-receptor interaction, calcium signaling pathway, cell adhesion molecules, and gap junction pathways (Fig. 5c, Table 4).

\section{Discussion}

In this study, 21 miRNAs related to $\mathrm{BC}$ prognosis were identified based on the expression levels in TCGA by univariate and multivariate Cox Regression analysis. Then, seven out of them were further isolated as the optimized prognostic gene signature and a MiR Score prognostic model was constructed based on these seven miRNAs (hsa-mir-1247, hsa-mir-1304, hsa-mir-1911, hsa-mir-204, hsa-mir-33b, hsa-mir-3934 and hsa-mir-526b), which presented a relative highly forecast ability for BC. In addition, age, lymphovascular invasion and MiR Score were identified as the independent prognostic factors in BC patients from TCGA. Furthermore, based on MiR Score prognostic model, several DEGs, such as WISP3 and $U N C 5 C$, as well as their related pathway, including cell-cell adhesion and neuroactive ligand-receptor interaction, were considered to be related to $\mathrm{BC}$ prognosis. Besides, we found $U N C 5 C$ is a potential target for hsamir-1911, hsa-mir-3934 and hsa-mir-526b through Targetscan, which belongs to the UNC-5 family of netrin receptors. Netrins are secreted proteins that direct axon extension and cell migration during neural development 


\section{a Training set $(\mathrm{N}=\mathbf{2 0 2})$}
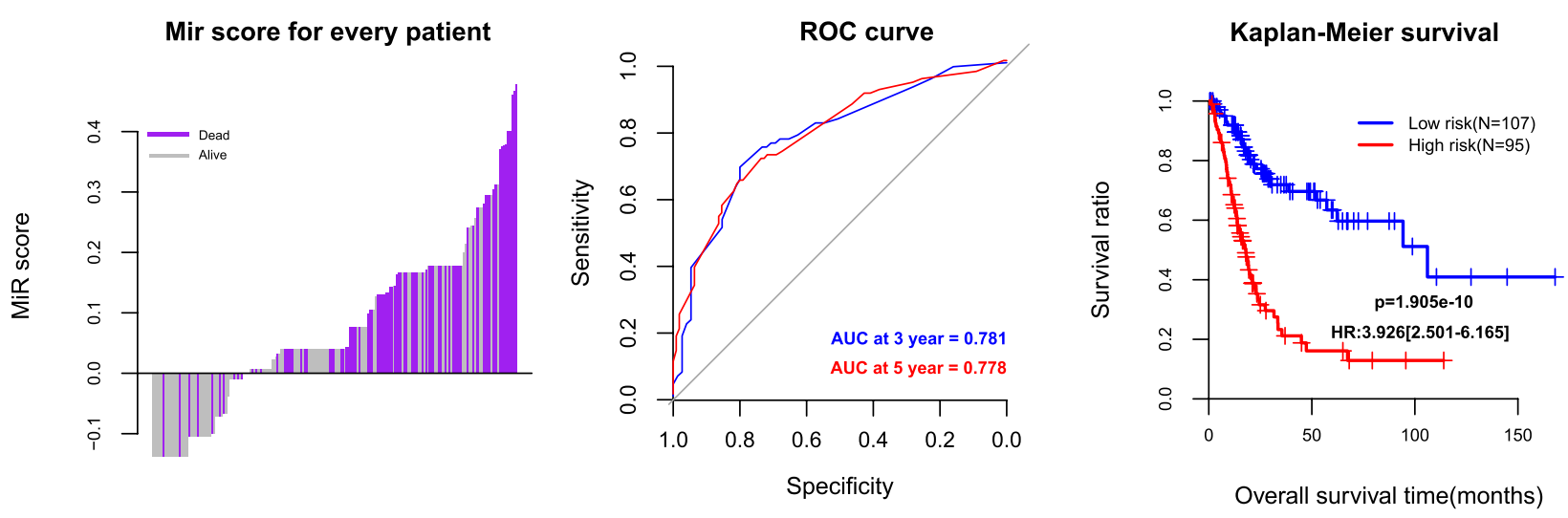

\section{b Validation set $(\mathrm{N}=\mathbf{2 0 3})$}
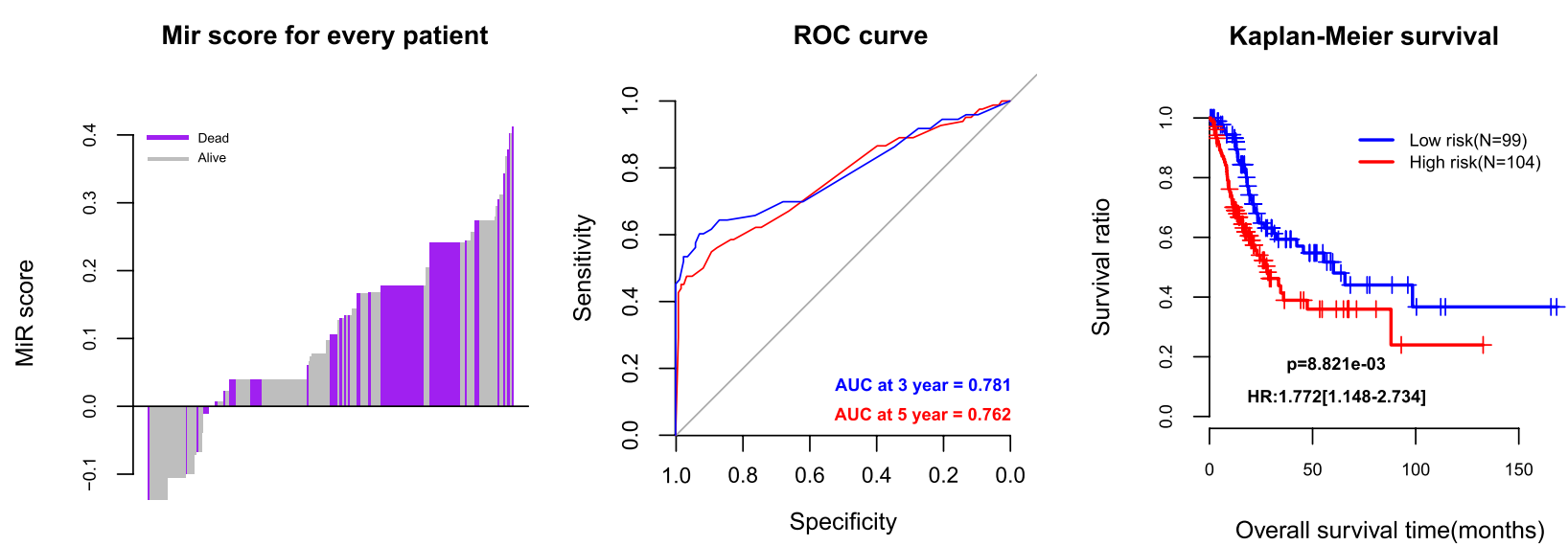

Fig. 3 a The sample distribution ofthe MiR Score in the training set (left), purple and gray bars represent samples of death and survival, respectively; ROC curve analysis of 3- and 5-year survival for MiR Score risk assessment model in the training set (middle); the K-M survival analysis of low- and high- risk groups in the training set (right), HR represents hazard ratio, and the number in parentheses indicates $95 \%$ confidence interval (CI). b The sample distribution of the MiR Score in the validation set (left), purple and gray bars represent samples of death and survival, respectively; ROC curve analysis of 3- and 5-year survival for MiR Score risk assessment model in the validation set (middle); the K-M survival analysis of low-and high-risk groups in the validation set (right), HR represents Hazard Ratio, and the number in parentheses indicates 95\% confidence interval (CI)

(NCBI, https://www.ncbi.nlm.nih.gov/). At present, studies have indicated that $U N C 5 C$ was associated with colorectal cancer [25, 26] and Alzheimer's Disease [27]. Thus, further studies are needed to identify the association between UNC5C and hsa-mir-1911, hsa-mir-3934 and hsa-mir-526b in BC patients.

The mining of a large amount of genetic data in various diseases have been enhanced due to the rapid technological advances in high-throughput sequencing and bioinformatics [28]. TCGA, as a public and available cancer genomic database, provides comprehensive data for different types of cancer, including mRNA expression data,
miRNA expression data, copy number variation, DNA methylation, and clinical information [29]. The data from TCGA have been effectively applied to improve diagnostic and therapeutic methods of cancers, as well as finally cancer prevention [29]. Thus, this study was also performed based on the miRNA expression profile data and clinical information of BC form TCGA. MiRNA expression profiles have been reported to predict the prognosis outcome of cancers [30]. Computationally, univariate and multivariate Cox Regression were the most common method to construct the prognostic models and screen prognostic factors [31]. In this study, the Cox Regression 
Table 3 Univariate and multivariate Cox Regression analysis of tumor samples in the training set and validation set

\begin{tabular}{|c|c|c|c|c|}
\hline \multirow[t]{2}{*}{ Clinical characteristics } & \multicolumn{2}{|l|}{ Uni-Cox regression } & \multicolumn{2}{|l|}{ Multi-Cox regression } \\
\hline & $\mathrm{HR}[95 \% \mathrm{Cl}]$ & $p$-value & $\mathrm{HR}[95 \% \mathrm{Cl}]$ & $p$-value \\
\hline \multicolumn{5}{|l|}{ Training set } \\
\hline Age (years, mean $\pm S D$ ) & $1.026[1.006-1.047]$ & $9.392 \times 10^{-3}$ & $1.016[1.011-1.430]$ & $2.034 \times 10^{-2}$ \\
\hline Gender (male/female) & $0.996[0.625-1.588]$ & $9.867 \times 10^{-1}$ & - & - \\
\hline Pathologic M (M0/M1/-) & $1.525[0.544-4.278]$ & $4.180 \times 10^{-1}$ & - & - \\
\hline Pathologic N (N0/N1/N2/N3/-) & 1.611 [1.289-2.013] & $1.615 \times 10^{-5}$ & $0.999[0.593-1.684]$ & $6.340 \times 10^{-1}$ \\
\hline Pathologic T (T1/T2/T3/T4/-) & $1.594[1.175-2.163]$ & $2.619 \times 10^{-3}$ & $0.858[0.486-1.516]$ & $9.980 \times 10^{-1}$ \\
\hline Pathologic stage (I/II/III/IV/-) & $1.661[1.288-2.142]$ & $6.357 \times 10^{-5}$ & $1.498[0.926-2.424]$ & $3.970 \times 10^{-1}$ \\
\hline Pathologic grade (High/low/-) & $3.164[0.439-22.77]$ & $2.27 \times 10^{-1}$ & - & - \\
\hline Radiotherapy (yes/no/-) & $0.948[0.233-3.863]$ & $9.402 \times 10^{-1}$ & - & - \\
\hline Lymphovascular invasion (yes/no/-) & $2.411[1.441-4.034]$ & $5.576 \times 10^{-4}$ & $1.709[0.893-3.274]$ & $1.050 \times 10^{-2}$ \\
\hline Recurrence (yes/no) & $1.621[0.986-2.667]$ & $5.473 \times 10^{-2}$ & - & - \\
\hline MiR score (high/low) & $3.926[2.500-6.165]$ & $1.905 \times 10^{-10}$ & 3.431 [1.919-6.130] & $3.180 \times 10^{-5}$ \\
\hline \multicolumn{5}{|l|}{ Validation set } \\
\hline Age (years, mean $\pm S D$ ) & $1.038[1.013-1.064]$ & $2.272 \times 10^{-3}$ & $1.069[1.0262-1.113]$ & $1.340 \times 10^{-3}$ \\
\hline Gender (male/female) & $1.275[0.808-2.011]$ & $2.955 \times 10^{-1}$ & - & - \\
\hline Pathologic M (M0/M1/-) & 23.27 [6.683-81.03] & $5.850 \times 10^{-1}$ & - & - \\
\hline Pathologic N (N0/N1/N2/N3/-) & $1.564[1.231-1.987]$ & $1.749 \times 10^{-3}$ & $1.652[0.854-3.195]$ & $1.359 E-01$ \\
\hline Pathologic T (T1/T2/T3/T4/-) & $1.985[1.421-2.773]$ & $5.099 \times 10^{-5}$ & $1.858[0.937-3.690]$ & $7.634 \times 10^{-2}$ \\
\hline Pathologic stage (I/II/III/IV/-) & $1.836[1.374-2.453]$ & $2.048 \times 10^{-5}$ & $0.668[0.276-1.617]$ & $3.710 \times 10^{-1}$ \\
\hline Pathologic grade (high/low/-) & $2.637[0.365-19.07]$ & $3.180 \times 10^{-1}$ & - & - \\
\hline Radiotherapy (yes/no/-) & $1.188[0.516-2.736]$ & $6.850 \times 10^{-1}$ & - & - \\
\hline Lymphovascular invasion (yes/no/-) & $2.335[1.338-4.074]$ & $2.119 \times 10^{-3}$ & $1.338[1.056-3.177]$ & $4.509 \times 10^{-2}$ \\
\hline Recurrence (yes/no) & $2.023[1.171-3.495]$ & $1.002 \times 10^{-2}$ & $1.002[0.468-2.144]$ & $9.960 \times 10^{-1}$ \\
\hline MiR score (high/low) & $1.772[1.148-2.734]$ & $8.821 \times 10^{-3}$ & $2.353[1.0702-5.176]$ & $3.328 \times 10^{-2}$ \\
\hline
\end{tabular}

Italic values indicate $p$ value $<0.05$

$M$ distant metastases, $N$ regional lymph node, $T$ tumor size, $H R$ hazard ratio

model based on the LASSO, a semi-parametric proportional hazards model, was applied. The availability of this model in survival analysis have been confirmed in recent studies [32, 33]. Similarly, in this study, the MiR Score prognostic model constructed by LASSO Cox Regression model showed a higher predictive ability both in training and validation sets. In addition, this study showed that age and lymphovascular invasion were independent prognostic factors in BC patients. Consistent with our results, previous studies have also demonstrated that age and lymphovascular invasion are associated with poor prognosis in BC patients [34], Notably, MiR Score was also been considered as an independent prognostic factor in $B C$ patients, which further showed that the MiR Score prognostic model had a significant predictive ability for $\mathrm{BC}$ prognosis.

In this study, the 7-miRNA signature was identified. Specifically, miR-1247, as a tumor suppressor, has been reported in several cancers, including lung cancer [35], hepatocellular cancer [36], and pancreatic cancer [37]. A recent study also has shown that miR-1247 inhibits cell proliferation and invasion through down-regulating its target gene RAB36 in BC [38]. MiR-204, miR-33b, and miR-526b are three reported miRNAs that function as tumor suppressor in cancers. Previous studies have suggested that miR-204 plays an inhibitory effect on cell invasion in gastric cancer cells [39] and non-small cell lung cancer [40]. MiR-33b has been reported to inhibit migration and invasion in osteosarcoma cells [41], melanoma cells [42], and lung adenocarcinoma cells [43]. In addition, miR-526b is revealed to have an inhibitory role in non-small cell lung cancer [44]. However, studies of these three miRNAs as well as miR-1304, miR-1911, and miR-3934 are rarely reported in BC. Therefore, it is important to further reveal the mechanism and prognostic significance of 7-miRNA signature in BC.

Furthermore, this study found that several DEGs, such as WISP3 and UNC5C, were closely associated with BC prognosis. WISP3, also known as cellular communication network factor 6 (CCN6), is a cysteine-rich and glycosylated signaling protein and key regulatory extracellular matrix component [45]. It is one member of the $\mathrm{CCN}$ 
a

Points

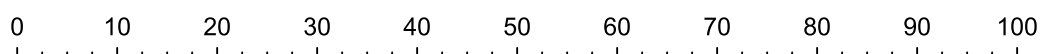

Age

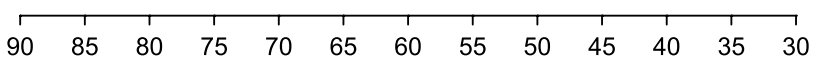

Invasion

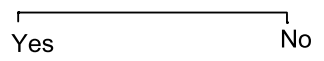

MiRScore

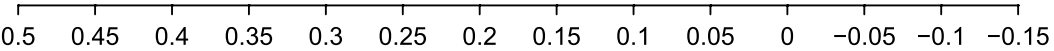

Total Points

\begin{tabular}{lllllllllllll}
\hline 0 & 20 & 40 & 60 & 80 & 100 & 120 & 140 & 160 & 180 & 200
\end{tabular}

3-year Survival Probability

$\begin{array}{lllllll}0.3 & 0.4 & 0.5 & 0.6 & 0.7 & 0.8 & 0.9\end{array}$

5-year Survival Probability

$\begin{array}{lllllllll}0.1 & 0.2 & 0.3 & 0.4 & 0.5 & 0.6 & 0.7 & 0.8 & 0.9\end{array}$

b

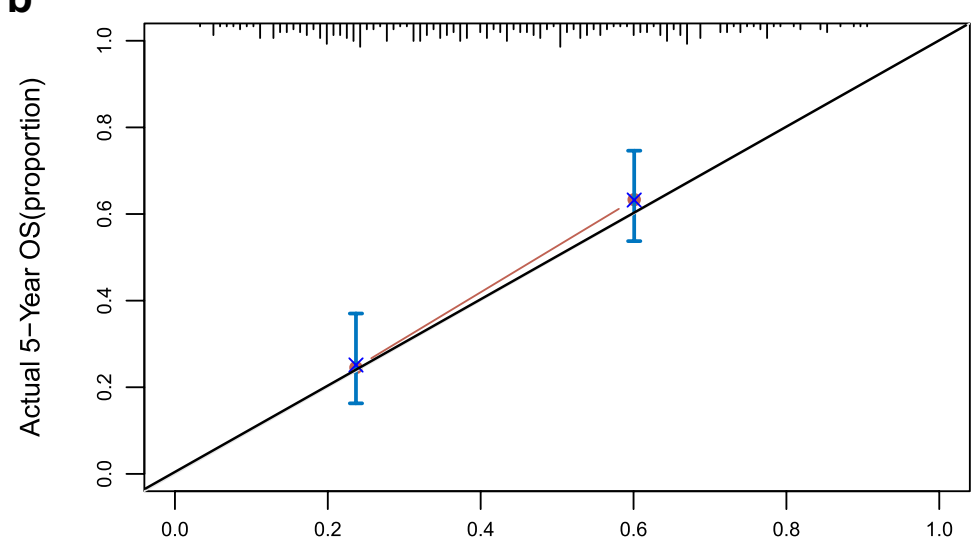

Nomogram-Predicted Probability of 5-Year OS

Fig. 4 a The nomogram of 3- and 5-year survival prediction models for these independent prognostic factors. b The nomogram of 5-year survival prediction compliant to actual 5-year survival

(Cyr61, CTGF, Nov) family which play important roles in several biological functions, including cell proliferation, adhesion, and invasion [45], which suggested the close relationship of WISP3 and cell-cell adhesion. Increasing evidences have demonstrated that WISP3 is abnormally expressed in cancers and play a contrary effect in cancer progression [46]. It is reported that WISP3 is overexpressed as an oncogene in ovarian carcinomas [47]. On the contrary, WISP3 is a tumor suppressor gene that inhibits cell proliferation in breast cancer [48]. Zeng et al. [49] has found that depletion of WISP3 notably inhibited the invasion of $\mathrm{BC}$ cells. Our data suggests that inhibition of WISP3 may be a therapeutic strategy for $\mathrm{BC}$. Recent study has found that WISP3 is up-regulated and promotes the cell proliferation and invasion in $\mathrm{BC}$ cells, which is consistent with our results. UNC5C (unc-5 netrin receptor $\mathrm{C}$ ) belongs to the netrin-1 receptor family, and plays key role in cell apoptotic process as a dependence receptor that may be involved in neuroactive ligand-receptor interaction [50]. Previous reports have suggested that $U N C 5 C$ has tumor suppressive effect in colorectal cancer through promoter methylation [51]. 


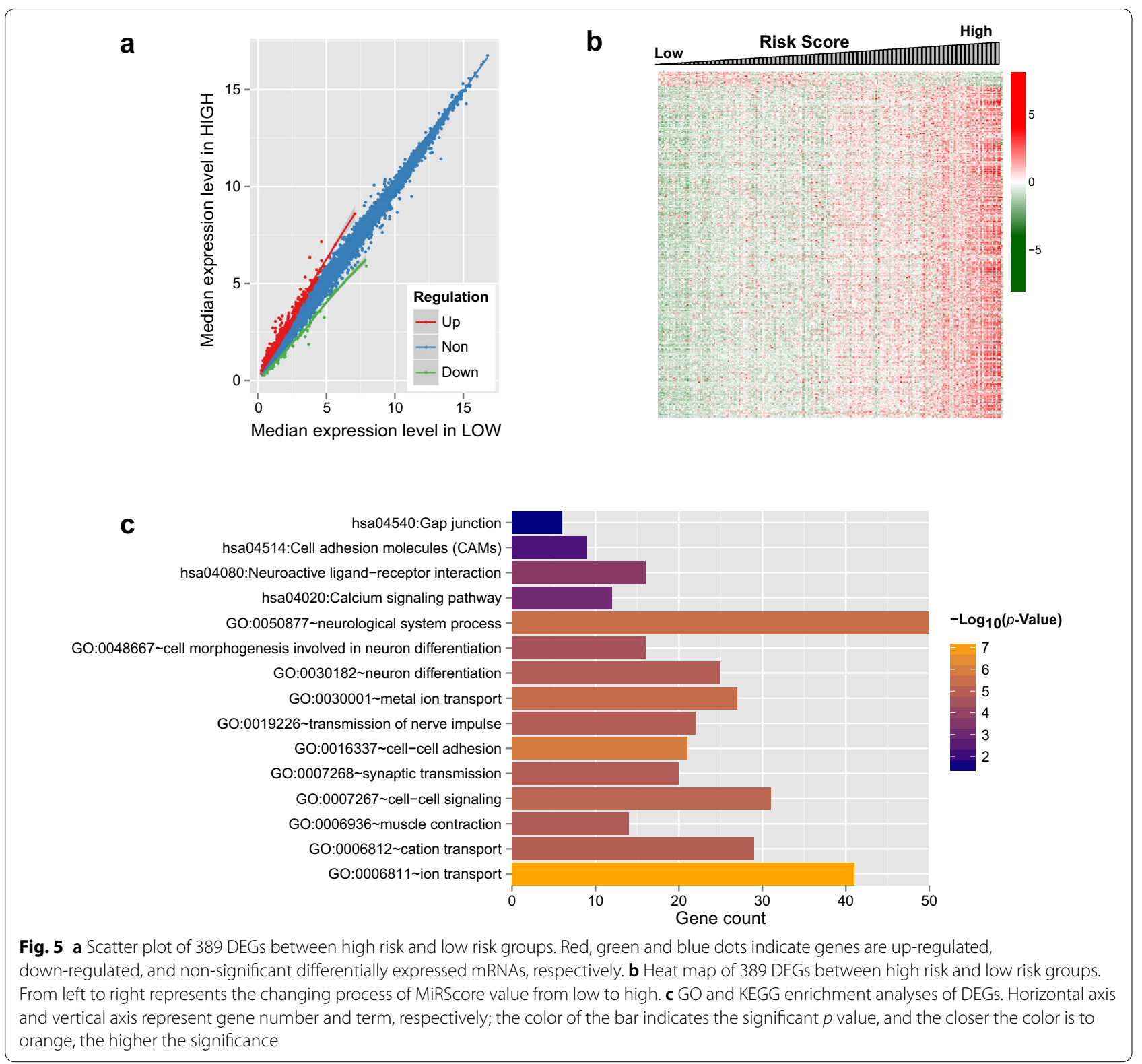

In addition, $U N C 5 C$ is reported to be downregulated due to specific genetic alterations and inhibits apoptosis of tumor cells by suppressing proapoptotic signals [52]. More recently, the receptors of UNC5 family have been revealed to be involved in the regulation of cell death processes in $\mathrm{BC}[53,54]$. However, there were still a number of limitations in the work. Functional validation was lacked for the feature genes obtained herein. Further investigations for these genes are required with substantial experiments. Nevertheless, this work provides novel insight into the pathogenesis of $\mathrm{BC}$.

\section{Conclusion}

In conclusion, the prognostic model based on the prognostic 7-miRNA signature presented a relatively promising predictive ability for $\mathrm{BC}$. The seven prognostic miRNAs may have clinical implications in BC prognosis. However, the prognostic significance of 7-miRNA 
Table 4 GO and KEGG enrichment analyses of DEGs

\begin{tabular}{|c|c|c|c|c|}
\hline Category & Term & Count & $p$-value & Genes \\
\hline \multirow[t]{11}{*}{ Biology process } & GO:0006811 ion transport & 41 & $5.51 \times 10^{-8}$ & SLC8A3, SLC36A2, GABRB3 \\
\hline & GO:0016337 cell-cell adhesion & 21 & $1.15 \times 10^{-6}$ & HCN4, SLC4A4, CAMK2A, \\
\hline & GO:0050877 neurological system process & 50 & $3.23 \times 10^{-6}$ & IGDCC3, OR10A3, GABRB3 \\
\hline & GO:0030001 metal ion transport & 27 & $3.97 \times 10^{-6}$ & $\mathrm{CPLX} 3, \mathrm{DRD} 2, \mathrm{OPRK} 1$ \\
\hline & GO:0007267 cell-cell signaling & 31 & $6.68 \times 10^{-6}$ & LIN7A, SFRP5, PENK \\
\hline & GO:0006812 cation transport & 29 & $1.09 \times 10^{-5}$ & SLC8A3, SLC36A2, DRD2 \\
\hline & GO:0019226 transmission of nerve impulse & 22 & $1.21 \times 10^{-5}$ & SLC24A5, CACNG4, KCNK2 \\
\hline & GO:0030182 neuron differentiation & 25 & $1.34 \times 10^{-5}$ & TUBB2B, RTN4RL1, PTPRZ1 \\
\hline & GO:0007268 synaptic transmission & 20 & $1.37 \times 10^{-5}$ & CPLX3, PLP1 \\
\hline & GO:0006936 muscle contraction & 14 & $1.69 \times 10^{-5}$ & ACTC1, MYBPC2, DRD2 \\
\hline & $\begin{array}{l}\text { GO:0048667 cell morphogenesis involved in neuron } \\
\text { differentiation }\end{array}$ & 16 & $2.87 \times 10^{-5}$ & RTN4RL1, DRD2, PTPRZ1 \\
\hline \multirow[t]{4}{*}{ KEGG pathway } & hsa04080:Neuroactive ligand-receptor interaction & 16 & $2.10 \times 10^{-4}$ & GPR156, CALCR, GABRB3 \\
\hline & hsa04020:Calcium signaling pathway & 12 & $9.18 \times 10^{-4}$ & SLC8A3, ATP2B2, ADRB3 \\
\hline & hsa04514:Cell adhesion molecules (CAMs) & 9 & 0.00562107 & NCAM1, NCAM2, CADM3 \\
\hline & hsa04540:Gap junction & 6 & 0.036085973 & ADCY2, TUBB2B, DRD2 \\
\hline
\end{tabular}

signature in $\mathrm{BC}$ should be futher confirmed in clinical study.

\section{Abbreviations}

BC: Bladder cancer; TCGA: The Cancer Genome Atlas; DEGs: Differentially expressed Genes; DEMs: Differentially expressed miRNAs; OS: Overall survival; miRNA: microRNA; FDR: False discovery rate; FC: Fold change; MiR Score: miRNA prognosis score; KEGG: Kyoto Encyclopedia of Genes and Genomes.

\section{Acknowledgements}

None.

\section{Authors' contributions}

YL and DYZ participated in the design of this study, and they both performed the statistical analysis. $\mathrm{HJX}$ and $\mathrm{YH}$ carried out the study and collected important background information. YS drafted the manuscript. All authors read and approved the final manuscript.

\section{Funding}

None.

\section{Availability of data and materials}

The raw data were collected and analyzed by the Authors, and are not ready to share their data because the data have not been published.

\section{Ethics approval and consent to participate}

Not applicable.

\section{Consent for publication}

Not applicable.

\section{Competing interests}

The authors declare no conflict of interests.

\section{Author details}

${ }^{1}$ Anesthesiology Department, Jilin Univ, China Japan Union Hosp, 126 Xiantai St, Changchun 130033, Jilin, People's Republic of China. ${ }^{2}$ Vascular Surgery Department, Jilin Univ, China Japan Union Hosp, 126 Xiantai St, Changchun 130033, Jilin, People's Republic of China. ${ }^{3}$ Department of Orthopedics, Jilin Univ, China Japan Union Hosp, 126 Xiantai St, Changchun 130033, Jilin, People's Republic of China. ${ }^{4}$ Department of Urology, Jilin Univ, China Japan
Union Hosp, 126 Xiantai St, Changchun 130033, Jilin, People's Republic of China.

Received: 11 August 2019 Accepted: 24 February 2020

Published online: 19 May 2020

\section{References}

1. Borkowska E, Konecki T, Pietrusiński M, Borowiec M, Jabłonowski ZJC. MicroRNAs which can prognosticate aggressiveness of bladder cancer. Cancers (Basel). 2019:11:E1551.

2. Bray F, Ferlay J, Soerjomataram I, Siegel RL, Torre LA, Jemal A. Global cancer statistics 2018: GLOBOCAN estimates of incidence and mortality worldwide for 36 cancers in 185 countries. CA Cancer J Clin. 2018;68:394-424

3. Cumberbatch MGK, Jubber I, Black PC, Esperto F, Figueroa JD, Kamat AM, et al. Epidemiology of bladder cancer: a systematic review and contemporary update of risk factors in 2018. Eur Urol. 2018;74:784-95.

4. Wang $P$, Wang $Y$, Hang B, Zou X, Mao J-H. A novel gene expression-based prognostic scoring system to predict survival in gastric cancer. Oncotarget. 2016;7:55343-51.

5. Flaig TW, Spiess PE, Agarwal N, Bangs R, Boorjian SA, Buyyounouski MK, et al. NCCN guidelines insights: bladder cancer, version 5.2018. J Natl Compr Canc Netw. 2018;16:1041-53.

6. Nagata M, Muto S, Horie S. Molecular biomarkers in bladder cancer: novel potential indicators of prognosis and treatment outcomes. Dis Mark. 2016:2016:8205836.

7. Acunzo M, Romano G, Wernicke D, Croce CM. MicroRNA and cancer-a brief overview. Adv Biol Regul. 2015:57:1-9.

8. Wu G, Wang F, Li K, Li S, Zhao C, Fan C, et al. Significance of TP53 mutation in bladder cancer disease progression and drug selection. PeerJ. 2019;7:e8261.

9. Kostopoulou ON, Holzhauser S, Lange BKA, Ohmayer A, Andonova T, Bersani C, et al. Analyses of FGFR3 and PIK3CA mutations in neuroblastomas and the effects of the corresponding inhibitors on neuroblastoma cell lines. Int J Oncol. 2019;55:1372-84.

10. Matsushita R, Seki N, Chiyomaru T, Inoguchi S, Ishihara T, Goto Y, et al. Tumour-suppressive microRNA-144-5p directly targets CCNE1/2 as potential prognostic markers in bladder cancer. Br I Cancer. 2015:113(2):282-9. 
11. Sakaguchi T, Yoshino H, Yonemori M, Miyamoto K, Sugita S, Matsushita R, et al. Regulation of ITGA3 by the dual-stranded microRNA-199 family as a potential prognostic marker in bladder cancer. Br J Cancer. 2018;118:e7.

12. Wang J, Zhang X, Wang L, Yang Y, Dong Z, Wang H, et al. MicroRNA-214 suppresses oncogenesis and exerts impact on prognosis by targeting PDRG1 in bladder cancer. PLOS ONE. 2015;10(2):e0118086.

13. Falzone L, Candido S, Salemi R, Basile MS, Scalisi A, McCubrey JA, et al. Computational identification of microRNAs associated to both epithelial to mesenchymal transition and NGAL/MMP-9 pathways in bladder cancer. Oncotarget. 2016;7:72758-66.

14. Vogt TJ, Gevensleben H, Dietrich J, Kristiansen G, Bootz F, Landsberg $J$, et al. Detailed analysis of adenosine $A 2$ a receptor $(A D O R A 2 A)$ and CD73 (5'-nucleotidase, ecto, NT5E) methylation and gene expression in head and neck squamous cell carcinoma patients. Oncoimmunology. 2018;7:1452579.

15. Ritchie ME, Phipson B, Wu D, Hu Y, Law CW, Shi W, et al. limma powers differential expression analyses for RNA-sequencing and microarray studies. Nucleic Acids Res. 2015;43:e47.

16. Eisen MB, Spellman PT, Brown PO, Botstein D. Cluster analysis and display of genome-wide expression patterns. Proc Natl Acad Sci USA. 1998;95:14863-8

17. Wang L, Cao C, Ma Q, Zeng Q, Wang H, Cheng Z, et al. RNA-seq analyses of multiple meristems of soybean: novel and alternative transcripts, evolutionary and functional implications. BMC Plant Biol. 2014;14:169.

18. Love MI, Huber W, Anders S. Moderated estimation of fold change and dispersion for RNA-seq data with DESeq2. Genome Biol. 2014;15:550.

19. Tibshirani R. The lasso method for variable selection in the Cox model. Sat Med. 1997;16:385-95.

20. Goeman JJ. L1 penalized estimation in the Cox proportional hazards model. Biometr J. 2010;52:70-84.

21. Camp RL, Dolled-Filhart M, Rimm DL. X-tile: a new bio-informatics tool for biomarker assessment and outcome-based cut-point optimization. Clin Cancer Res. 2004;10:7252-9.

22. Chen L, Wen Y, Zhang J, Sun W, Lui VWY, Wei Y, et al. Prediction of radiotherapy response with a 5-microRNA signature-based nomogram in head and neck squamous cell carcinoma. Cancer Med. 2018;7:726-35.

23. Ashburner M, Ball CA, Blake JA, Botstein D, Butler H, Cherry JM, et al. Gene ontology: tool for the unification of biology. The Gene Ontology Consortium. Nat Genet. 2000;25:25-9.

24. Kanehisa M, Goto S. KEGG: Kyoto encyclopedia of genes and genomes. Nucleic Acids Res. 2000;28:27-30.

25. Guroo SA, Malik AA, Afroze D, Ali S, Pandith AA, Yusuf A. Significant pattern of promoter hypermethylation of UNC5C gene in colorectal cancer and its implication in late stage disease. Asian Pac J Cancer Prev. 2018;19:1185-8.

26. Wu J, Wang G, He B, Chen X, An Y. Methylation of the UNC5C gene and its protein expression in colorectal cancer. Tumour Biol. 2017;39:1010428317697564.

27. Sun J-H, Wang H-F, Zhu X-C, Yu W-J, Tan C-C, Jiang T, et al. The impact of UNC5C genetic variations on neuroimaging in Alzheimer's disease. Mol Neurobiol. 2016;53:6759-67.

28. da Huang W, Sherman BT, Lempicki RA. Systematic and integrative analysis of large gene lists using DAVID bioinformatics resources. Nat Protoc. 2009;4:44-57.

29. Lambert C, Braxton C, Charlebois R, Deyati A, Duncan P, La Neve F, et al. Considerations for Optimization of High-throughput sequencing bioinformatics pipelines for virus detection. Viruses. 2018;10:e528.

30. Hutter C, Zenklusen JC. The Cancer Genome Atlas: creating lasting value beyond its data. Cell. 2018;173:283-5.

31. Schetter AJ, Leung SY, Sohn JJ, Zanetti KA, Bowman ED, Yanaihara N, et al. MicroRNA expression profiles associated with prognosis and therapeutic outcome in colon adenocarcinoma. JAMA. 2008;299:425-36.

32. Bao Z, Zhang W, Dong D. A potential prognostic IncRNA signature for predicting survival in patients with bladder urothelial carcinoma. Oncotarget. 2017;8:10485-97.

33. Ching T, Zhu $X$, Garmire LX. Cox-nnet: an artificial neural network method for prognosis prediction of high-throughput omics data. PLoS Comput Biol. 2018;14:e1006076.
34. Liang R, Wang M, Zheng G, Zhu H, Zhi Y, Sun Z. A comprehensive analysis of prognosis prediction models based on pathway-level, gene-level and clinical information for glioblastoma. Int J Mol Med. 2018;42:1837-46.

35. Nielsen ME, Shariat SF, Karakiewicz PI, Lotan Y, Rogers CG, Amiel GE, et al. Advanced age is associated with poorer bladder cancer-specific survival in patients treated with radical cystectomy. Eur Urol. 2007;51:699-708.

36. Chu Y, Fan W, Guo W, Zhang Y, Wang L, Guo L, et al. miR-1247-5p functions as a tumor suppressor in human hepatocellular carcinoma by targeting Wnt3. Oncol Rep. 2017;38:343-51.

37. Shi S, Lu Y, Qin Y, Li W, Cheng H, Xu Y, et al. miR-1247 is correlated with prognosis of pancreatic cancer and inhibits cell proliferation by targeting neuropilins. Curr Mol Med. 2014;14:316-27.

38. Zhu Y, Liang S, Pan H, Cheng Z, Rui X. Inhibition of miR-1247 on cell proliferation and invasion in bladder cancer through its downstream target of RAB36. J Biosci. 2018;43:365-73.

39. Zhang L, Wang X, Chen P. MiR-204 down regulates SIRT1 and reverts SIRT1-induced epithelial-mesenchymal transition, anoikis resistance and invasion in gastric cancer cells. BMC Cancer. 2013;13:290.

40. Shi L, Zhang B, Sun X, Lu S, Liu Z, Liu Y, et al. MiR-204 inhibits human NSCLC metastasis through suppression of NUAK1. Br J Cancer. 2014;111:2316-27.

41. Xu N, Li Z, Yu Z, Yan F, Liu Y, Lu X, et al. MicroRNA-33b suppresses migration and invasion by targeting c-Myc in osteosarcoma cells. PLoS ONE. 2014;9:e115300.

42. Zhang P, Bai H, Liu G, Wang H, Chen F, Zhang B, et al. MicroRNA-33b, upregulated by EF24, a curcumin analog, suppresses the epithelial-tomesenchymal transition (EMT) and migratory potential of melanoma cells by targeting HMGA2. Toxicol Lett. 2015;234:151-61.

43. Qu J, Li M, An J, Zhao B, Zhong W, Gu Q, et al. MicroRNA-33b inhibits lung adenocarcinoma cell growth, invasion, and epithelial-mesenchymal transition by suppressing Wnt/ $\beta$-catenin/ZEB1 signaling. Int J Oncol. 2015;47:2141-52

44. Zhang Z-Y, Fu S-L, Xu S-Q, Zhou X, Liu X-S, Xu Y-J, et al. By downregulating Ku80, hsa-miR-526b suppresses non-small cell lung cancer. Oncotarget. 2015:6:1462-77.

45. Holbourn KP, Acharya KR, Perbal B. The CCN family of proteins: structurefunction relationships. Trends Biochem Sci. 2008;33:461-73.

46. Gery S, Xie D, Yin D, Gabra H, Miller C, Wang H, et al. Ovarian carcinomas: CCN genes are aberrantly expressed and CCN1 promotes proliferation of these cells. Clin Cancer Res. 2005;11:7243-54.

47. Gao H, Yin F-F, Guan D-X, Feng Y-X, Zheng Q-W, Wang X, et al. Liver cancer: WISP3 suppresses hepatocellular carcinoma progression by negative regulation of $\beta$-catenin/TCF/LEF signalling. Cell Prolif. 2019;52:e12583.

48. Kleer CG, Zhang Y, Pant Q, Merajver SD. WISP3 (CCN6) is a secreted tumorsuppressor protein that modulates IGF signaling in inflammatory breast cancer. Neoplasia. 2004;6:179-85.

49. Zeng J, Liao Y, Zhou J, Yang G, Ding K, Zhang X. Role of WISP3 siRNA in proliferation, apoptosis and invasion of bladder cancer cells. Int J Clin Exp Med. 2015;8:12792-800.

50. Arakawa H. Netrin-1 and its receptors in tumorigenesis. Nat Rev Cancer. 2004:4:978-87.

51. Hibi K, Mizukami H, Shirahata A, Goto T, Sakata M, Sanada Y. Aberrant methylation of the netrin-1 receptor genes UNC5C and DCC detected in advanced colorectal cancer. World J Surg. 2009;33:1053-7.

52. Mazelin L, Bernet A, Bonod-Bidaud C, Pays L, Arnaud S, Gespach C, et al. Netrin-1 controls colorectal tumorigenesis by regulating apoptosis. Nature. 2004:431:80-4

53. Zhu Y, Yu M, Chen Y, Wang Y, Wang J, Yang C, et al. DNA damage-inducible gene, UNC5A, functions as a tumor-suppressor in bladder cancer. Tumor Biol. 2014;35:6887-91.

54. Zhu Y, Yu M, Chen Y, Wang Y, Wang J, Yang C, et al. Down-regulation of UNC5D in bladder cancer: UNC5D as a possible mediator of cisplatin induced apoptosis in bladder cancer cells. J Urol. 2014;192:575-82.

\section{Publisher's Note}

Springer Nature remains neutral with regard to jurisdictional claims in published maps and institutional affiliations. 